\title{
The responses of serum transferrin receptors to iron supplements in subjects with iron-deficiency erythropoiesis and iron-deficiency anaemia
}

\author{
Xiao-Ming Lin*, Wei Tian, Le Ma, Zhu Long, Juan Zhang, Xiao-Yi Shen and Xiao-Peng Zhang \\ Department of Nutrition and Food Hygiene, School of Public Health, Peking University, 38 Xueyuan Road, Beijing, 100083, China \\ (Received 5 December 2006 - Revised 12 June 2007 - Accepted 13 June 2007)
}

We aimed to study the response of serum transferrin receptors (sTfR) to Fe supplementation in women of childbearing age with Fe-deficiency erythropoiesis (IDE) and Fe-deficiency anaemia (IDA). Primary screening was performed in 942 women ranging in age from 18 to 45 years. After Fe-related biochemical indices such as serum ferritin, $\mathrm{Zn}$ protoporphyrin and $\mathrm{Hb}$ were determined, the subjects were divided into four groups: normal, Fe store depletion, IDE and IDA. A total of 131 women were randomly selected from the normal, IDE and IDA groups. Subsequently, seventy-six women with IDE and IDA were given various doses of Fe (14 mg/d for IDE; $28 \mathrm{mg} / \mathrm{d}$ for IDA) with ferrous L-threonate capsules for twelve consecutive weeks. After receiving Fe supplements, the levels of Fe and sTfR were determined at weeks $0,3,6,9$ and 12.The levels of sTfR in women of childbearing age with IDE and IDA were significantly higher than those in the normal group. After receiving Fe supplements, the levels of sTfR were significantly decreased in women of childbearing age with IDE and IDA, while the levels of serum ferritin were significantly increased. In conclusion, STfR can be used as a reliable indicator for assessing the efficacy of Fe supplements.

Iron supplementation: Serum transferrin receptors: Reproductive-age women: Serum ferritin

Fe-deficiency anaemia (IDA) is a public health problem throughout the world, particularly in developing countries. Fe deficiency is especially prevalent among infants, children, pregnant women and women of childbearing age. The transition from normal levels to the development of IDA involves two sequential processes: $\mathrm{Fe}$ store depletion and $\mathrm{Fe}$ deficiency erythropoiesis (IDE). There are no additional physiological phenomena associated with the development of Fe store depletion and IDE, so both are classified as subclinical $\mathrm{Fe}$ deficiency ${ }^{1}$. After exhaustion of the stored $\mathrm{Fe}$ compartment, a subsequent depletion in the functional $\mathrm{Fe}$ compartment, IDE and IDA, begins ${ }^{2}$. Although the assessment of $\mathrm{Fe}$ status is notoriously difficult because of the lack of a sensitive index for screening specifically for Fe deficiency, insufficient $\mathrm{Fe}$ intake from food and poor Fe bioavailability are normally held responsible for IDA $^{3}$. The most widely used benchmarks to study IDA in the general population are the use of measurements of $\mathrm{Hb}$ and serum ferritin (SF), but this practice has been criticised. Therefore, it is critical to find a sensitive, specific and applicable biochemical marker to determine the magnitude of early-stage $\mathrm{Fe}$ deficiency. Currently, the concentration of serum transferrin receptors (sTfR) has been reported useful in the diagnosis of Fe deficiency. It is superior to the traditional laboratory methods for identifying $\mathrm{Fe}$ status because the sTfR levels do not appear to be influenced by inflammation and infection and sTfR levels have been reported to have low biological variability $^{4-6}$.
However, sTfR studies mainly concentrate on its efficacy in the diagnosis of Fe deficiency. Little is known about the change trends and characteristics of sTfR levels during Fe supplementation. In the present study, we examine the responses of sTfR to Fe supplementation, discuss the dynamic changes characteristic of each stage, and provide evidence to show that sTfR can be used as a reliable indicator for assessing the efficacy of Fe supplementation.

\begin{abstract}
Subjects and methods
Subjects

The study population originally consisted of 942 women, ranging in age from 18 to 45 years. They were recruited using advertisements and lectures in the suburban areas of Beijing and the Langfang City of Hebei Province and were taken from the lower or middle socio-economic stratum. Their levels of education and income, marital status and occupation were recorded at the time of their recruitment. A detailed clinical and anthropometric examination was conducted on all participants. Women with a history of smoking, alcohol use, haematological diseases, asthma, diseases of the musculoskeletal system or recent infections or recent pregnancy (within the last year) and women taking $\mathrm{Fe}$ supplements by mouth were excluded from the study. The medical ethics committee of Peking University approved the protocol. All participants provided signed consent forms after the nature of the study was provided to them.
\end{abstract}


Study design

The Fe status of all subjects was assessed at the onset of the present study by determining $\mathrm{SF}, \mathrm{Zn}$ protoporphyrin (ZPP) and $\mathrm{Hb}$ levels in venous blood samples. According to the current standard of evaluating levels of $\mathrm{Fe}$, the subjects were divided into four groups: normal, Fe store depletion, IDE and IDA $^{7}$. The evaluation standards for Fe-deficiency are: $\mathrm{Fe}$ store depletion, $\mathrm{Hb}>120 \mathrm{~g} / \mathrm{l}, \mathrm{ZPP}<1.4 \mu \mathrm{g} / \mathrm{g}, \mathrm{SF}<20 \mu \mathrm{g} / \mathrm{l}$; IDE $, \quad \mathrm{Hb}>120 \mathrm{~g} / \mathrm{l}, \quad \mathrm{ZPP}>1.4 \mu \mathrm{g} / \mathrm{g}, \quad \mathrm{SF}<20 \mu \mathrm{g} / \mathrm{l} ; \quad$ IDA, $\mathrm{Hb}<120 \mathrm{~g} / \mathrm{l}, \mathrm{ZPP}>1.4 \mu \mathrm{g} / \mathrm{g}, \mathrm{SF}<20 \mu \mathrm{g} / \mathrm{l}^{8}$.

A total of 131 women were randomly selected from those with normal ( $n$ 55), IDE ( $n$ 31) and IDA ( $n$ 45) levels to determine sTfR. Among them, women with various stages of $\mathrm{Fe}$ deficiency $(n$ 76) were included in the 12-week intervention study. Because low Fe storage does not equal Fe deficiency, and there are no additional physiological phenomena in the Fe store depletion stage, the women with Fe store depletion were excluded from the intervention study.

\section{Laboratory analysis}

After fasting overnight, blood specimens $(4.0 \mathrm{ml})$ were obtained from the subjects by certified laboratory technicians to determine the parameters associated with $\mathrm{Fe}$ status. Blood specimens were processed at a local examination centre and sent to a laboratory in Beijing. Whole-blood specimens $(0.02 \mathrm{ml})$ were added to light-avoided sterile heparin-anticoagulant tubes to determine ZPP levels using an accurate, well-calibrated ZPP haematofluorometer (microfluorescence method, ZPP haematofluorometer model 3800; Guangzhou Kangda Industry Co. Ltd, Guangzhou, Guangdong, China).

Whole-blood specimens $(0.02 \mathrm{ml})$ were added to methaemoglobin cyanide dilution and $\mathrm{Hb}$ concentration was determined (methaemoglobin cyanide method, spectrophotometer model 724; Shanghai no. 3 Analysis Instrument Factory, Shanghai, China, and table-top multi-application oven-controlled oscillator model HZ-881K; Taicang Scientific Experimental Instrument Factory, Taicang, Jiangsu, China). The remaining blood was centrifuged (Freeze Centrifuge model DDL-5; Shanghai An Ting Scientific Instrument Factory, Shanghai, China) to isolate serum that was stored at $-70^{\circ} \mathrm{C}$ for determination of the SF and sTfR. The SF was determined by the radioimmunological method (intelligent $\gamma$ RIA measurement instrument, model SN-695B; RuiHuan Instrument Factory of Shanghai Nuclear Research Institute, Shanghai, China) using a $\left[{ }^{125}\right.$ I] serum ferritin kit (The Atomic Energy Institute, Beijing, China). The sTfR was determined using a double antibody sandwich ELISA kit (R\&D Systems, Minneapolis, MN, USA, and enzyme calibration enzyme-linked apparatus model 450; Bio-Rad Co., Hercules, CA, USA).

All members of the study team successfully completed a training programme on the aims of the study and the specific methods used. The biological measurements were standardised among laboratories according to the criteria of Peking University.

\section{Iron supplementation}

In the study, the women in the IDE and IDA groups were given different doses of $\mathrm{Fe}$ supplements for 12 weeks ( $14 \mathrm{mg} / \mathrm{d}$ for IDE; $28 \mathrm{mg} / \mathrm{d}$ for IDA) with ferrous L-threonate capsules that were provided by Beijing Juneng New Technique Co. Ltd (Beijing, China).

The Food and Drug Administration of China certified that the capsules contained $7 \mathrm{mg} F$ and $30 \mathrm{mg}$ vitamin $\mathrm{C}$. The women were instructed to take the capsules between meals, with no change in their diets, and were asked to keep a record, on cards, of when the capsules had been taken. After taking $\mathrm{Fe}$ supplements, the parameters reflecting $\mathrm{Fe}$ status and sTfR levels were determined at weeks $0,3,6,9$ and 12 .

\section{Statistical analyses}

The $t$ test and variance analysis were used to compare the differences in all of the parameters among the three groups. Univariate correlation and multiple linear regression were used to analyse the relationship among the variables. Trends in STfR and sTfR:SF over time were analysed using the $\chi^{2}$ test for trends. SF and sTfR showed skewness in distribution, so a $\log$ transformation was performed first to acquire normal distribution, and statistical analysis was then carried out. All the analyses were conducted with SPSS 10.0 for Windows (SPSS Inc., Chicago, IL, USA).

\section{Results}

Iron status and serum transferrin receptors levels in subjects

The results of sTfR and Fe-related biochemical indices before $\mathrm{Fe}$ supplementation are summarised in Table 1. Compared with the group of women with normal Fe levels, the sTfR, sTfR:SF, Hb, $\mathrm{ZPP}$ and SF levels differed significantly in Fe-deficient women $(P<0.05)$. Furthermore, the sTfR, sTfR:SF, $\mathrm{Hb}$ and ZPP levels in the IDA group differed significantly from the IDE group $(P<0.05)$. The sTfR and sTfR:SF levels were 2.28 and 3.91 times higher than those of normal controls, respectively.

\section{Correlation between serum transferrin receptors and iron- related biochemical indices}

To determine if there might be an independent association between sTfR concentration and Fe-related biochemical indices, we examined the relationship of sTfR with concentrations of $\mathrm{Hb}, \mathrm{SF}$ and ZPP. Among seventy-six subjects, there were significant negative correlations of STfR with $\mathrm{Hb}$ and SF and a significant positive correlation with ZPP. The correlation with ZPP was the most significant (Table 2). If sTfR was taken as a dependent variable and $\mathrm{Hb}, \mathrm{ZPP}$ and SF were taken as independent variables to construct a multiple regression model, the multiple correlation coefficient was $0 \cdot 821$. ZPP was a significant variable and the correlation coefficient was 0.713 . When the insignificant variable SF was eliminated and another regression model was constructed, the correlation coefficients of sTfR with ZPP and $\mathrm{Hb}$ were 0.722 and -0.181 , respectively.

\section{Dynamic changes of serum transferrin receptors in various stages during iron supplementation}

Fe supplementation was given to the IDE and IDA groups. Table 3 shows a significant treatment effect in Fe-deficient women over time. After the 12 weeks of Fe supplementation, sTfR levels were significantly changed. The $\chi^{2}$ test for trend 
Table 1. Values of serum transferrin receptor (sTfR) and iron-related biochemical indices in iron-deficient and normal subjects before iron supplementation (Mean values and standard deviations)

\begin{tabular}{|c|c|c|c|c|c|c|c|c|c|c|c|c|c|}
\hline \multirow[b]{2}{*}{ Group } & \multirow[b]{2}{*}{$n$} & \multicolumn{2}{|c|}{ Age (years) } & \multicolumn{2}{|c|}{$\mathrm{Hb}(\mathrm{g} / \mathrm{l})$} & \multicolumn{2}{|c|}{$\mathrm{ZPP}(\mu \mathrm{mol} / \mathrm{l})$} & \multicolumn{2}{|c|}{ SF $(\mu \mathrm{g} / \mathrm{l})$} & \multicolumn{2}{|c|}{ STfR (nmol/l) } & \multicolumn{2}{|c|}{ sTfR:SF } \\
\hline & & Mean & SD & Mean & SD & Mean & SD & Mean & SD & Mean & SD & Mean & SD \\
\hline Normal & 55 & 36.34 & 6.69 & 142.50 & 6.90 & 0.44 & 0.15 & $51 \cdot 20$ & $18 \cdot 11$ & 18.57 & 4.48 & $10 \cdot 86$ & 2.57 \\
\hline IDE & 31 & 34.96 & $7 \cdot 12$ & $125 \cdot 30^{*}$ & $12 \cdot 10$ & $2 \cdot 32^{*}$ & 1.53 & $10.58^{*}$ & $4 \cdot 21$ & $27 \cdot 73^{\star}$ & 9.85 & $27 \cdot 21^{*}$ & 14.90 \\
\hline IDA & 45 & 35.92 & 6.02 & $102 \cdot 90^{*} \dagger$ & $15 \cdot 20$ & $3.75^{\star} \dagger$ & 2.04 & $11 \cdot 19^{*}$ & 4.29 & $42 \cdot 31^{*} \dagger$ & 20.12 & $42 \cdot 46^{*} \dagger$ & $29 \cdot 31$ \\
\hline
\end{tabular}

ZPP, Zn protoporphyrin; SF, serum ferritin; IDE, Fe-deficiency erythropoiesis; IDA, Fe-deficiency anaemia.

${ }^{*}$ Mean value was significantly different from that for normal women $(P<0.05)$.

$\dagger$ Mean value was significantly different from that for the IDE group $(P<0.05)$.

Table 2. Relationships between serum transferrin receptor and iron-related biochemical indices haemoglobin, serum ferritin (SF) and zinc protoporphyrin (ZPP) in subjects with irondeficiency erythropoiesis and iron-deficiency anaemia

\begin{tabular}{llll}
\hline & $n$ & Correlation coefficient & $P$ \\
\hline $\mathrm{Hb}$ & 76 & -0.561 & $<0.001$ \\
$\mathrm{SF}$ & 76 & -0.463 & $<0.001$ \\
ZPP & 76 & 0.811 & $<0.001$ \\
\hline
\end{tabular}

showed statistically significant differences in sTfR levels at weeks $0,3,6,9$ and $12\left(P<0 \cdot 001\right.$ by $\chi^{2}$ test for trend). In the IDE stage, the sTfR level did not show significant change in the first 3 weeks. It decreased gradually after 3 weeks. sTfR concentration at week 6 was significantly lower than that at week $3(P<0 \cdot 01)$, but there was no significant change from week 6 to week 12 and the sTfR level reached the normal range by the end of the study. In the IDA stage, the sTfR level decreased rapidly in the first 3 weeks. At weeks 3, 6 and 9, sTfR concentrations showed greatly significant changes and tended to reach normal levels at week 12 when Fe supplementation was complete. The details of these results are shown in Table 3 and Fig. 1.

Dynamic changes of serum transferrin receptor:serum ferritin ratio during iron supplementation

The change trend of sTfR:SF values was basically consistent with sTfR $\left(P<0.001\right.$ by $\chi^{2}$ test for trend). In the IDA stage,
sTfR:SF values had a significantly greater decrease at weeks 3,6 and 12. However, sTfR concentration at that stage showed no significant changes at weeks 3 and 12 (Table 3). The details of these results are shown in Table 4 and Fig. 2.

\section{Discussion}

Transferrin receptor is a type of transmembrane glycoprotein expressed on virtually all cells. It can bind to Fe-carrying transferrin in plasma specifically, and transfers Fe into cells through receptor-induced endocytosis 9 . Its classic function is to regulate the uptake of $\mathrm{Fe}$ into the cells from circulating transferrin. sTfR present in serum is a truncated fragment of the membrane transferrin receptor and the concentration reflects tissue expression ${ }^{10}$, so sTfR expression is regulated by body Fe status. Fe deficiency is associated with increased sTfR levels and elevated Fe storage is associated with reduced sTfR concentration. Recently, sTfR has been proposed as a reliable parameter for assessing $\mathrm{Fe}$ status and erythropoietic activity $^{5,11}$. In the present study, compared with subjects with normal levels of Fe, sTfR levels in women with IDE and IDA were significantly increased. With progressive Fe deficiency, the concomitant changes of sTfR appeared to be consistent with earlier studies ${ }^{11}$ with their values greatly different from those with normal levels. In the present study, we found that STfR was significantly increased with progressive Fe deficiency and the sTfR levels in the IDE and IDA groups were 1.49 and 2.28 times as high as those in the normal group, which suggests that STfR is a sensitive parameter to detect $\mathrm{Fe}$ deficiency, even for subclinical $\mathrm{Fe}$

Table 3. Dynamic changes of serum transferrin receptor (sTfR) in subjects with iron-deficiency erythropoiesis and iron-deficiency anaemia during iron supplementation

(Mean values and standard deviations)

\begin{tabular}{|c|c|c|c|c|c|c|c|c|c|c|}
\hline \multirow[b]{3}{*}{ Week } & \multicolumn{5}{|c|}{ Fe-deficiency erythropoiesis } & \multicolumn{5}{|c|}{ Fe-deficiency anaemia } \\
\hline & \multirow[b]{2}{*}{$n$} & \multicolumn{2}{|c|}{ STfR (nmol/l) } & \multirow[b]{2}{*}{ sTfR† } & \multirow[b]{2}{*}{ sTfR $\ddagger$} & \multirow[b]{2}{*}{$n$} & \multicolumn{2}{|c|}{ STfR (nmol/l) } & \multirow[b]{2}{*}{ sTfR† } & \multirow[b]{2}{*}{ sTfR $\neq$} \\
\hline & & Mean & SD & & & & Mean & SD & & \\
\hline 0 & 31 & $27 \cdot 73$ & $9 \cdot 85$ & 1.00 & 1.00 & 45 & $42 \cdot 31$ & $20 \cdot 12$ & 1.00 & 1.00 \\
\hline 3 & 31 & $26 \cdot 03$ & 8.45 & 0.94 & 0.94 & 45 & $29 \cdot 15^{\star \star}$ & $14 \cdot 10$ & 0.69 & 0.69 \\
\hline 6 & 31 & $21 \cdot 17^{\star \star}$ & 5.49 & 0.76 & 0.81 & 45 & $25 \cdot 22^{\star}$ & $9 \cdot 91$ & 0.60 & 0.87 \\
\hline 9 & 31 & $19 \cdot 31$ & $5 \cdot 07$ & 0.70 & 0.91 & 45 & $19 \cdot 83^{\star *}$ & $6 \cdot 01$ & 0.47 & 0.79 \\
\hline 12 & 31 & $17 \cdot 74$ & 4.42 & 0.64 & 0.92 & 45 & $19 \cdot 18$ & $5 \cdot 21$ & 0.45 & 0.97 \\
\hline
\end{tabular}

Mean value was significantly different from that for the previous week: ${ }^{\star} P<0.05,{ }^{\star \star} P<0.01$

$\dagger$ Ratio of values of sTfR index in groups to those at week 0 (relative ratio with fixed base).

$\ddagger$ Ratio of values of sTfR index in groups to those of the previous week (link-relative ratio) 


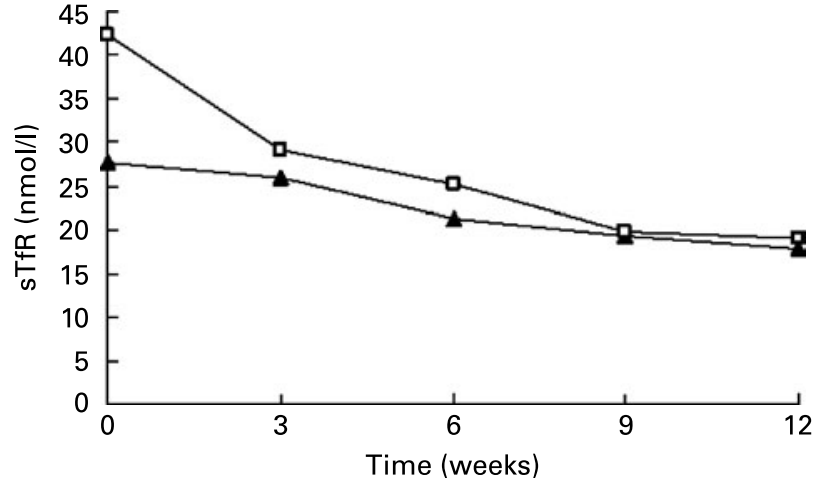

Fig. 1. Values of serum transferrin receptor (sTfR) in subjects with Fedeficiency erythropoiesis $(\boldsymbol{\Lambda} ; n$ 31) and Fe-deficiency anaemia $(\square ; n$ 45) during Fe supplementation. Values are means.

deficiency. However, the sTfR increment range in the present study was smaller than some other studies. For example, Kogho et al. studied forty-one IDA patients, and their sTfR levels were $2 \cdot 8$ times higher than the normal value ${ }^{12}$. Similarly, Flowers et al. conducted a study involving nineteen IDA patients, and the sTfR levels were $3 \cdot 2$ times higher than those in the normal group ${ }^{13}$. Such differences might be due to the different body Fe-deficiency levels among the subjects in the present study and theirs. The subjects in Kogho's and Flowers' study were hospital patients with very severe anaemia, while our subjects were selected from a 'normal' population and most had mild anaemia. In experiments with animals, the sTfR levels in rats with severe IDA were several times higher than those in the normal control group ${ }^{14}$.

Through statistical analysis, highly significant direct associations were observed between sTfR concentration and these indices. STfR had a negative correlation with $\mathrm{Hb}$ and SF and a positive correlation with ZPP. Its correlation with ZPP was the most significant. We further examined this relationship in multivariate linear regression models of sTfR by including $\mathrm{Hb}, \mathrm{ZPP}$ and SF. In these models, ZPP was the best indicator of sTfR, possibly because both sTfR and ZPP reflect functional $\mathrm{Fe}$ status and erythropoietic activity, in contrast with $\mathrm{Hb}$ and SF. Hb reflects severe Fe deficiency and SF reflects $\mathrm{Fe}$ storage. Both of them show low sensitivity and specificity. This might explain the apparently significant association that we observed between sTfR with ZPP. Collectively, sTfR is highly correlated with functional Fe-deficiency biochemical indices. It can be used as an index to evaluate functional $\mathrm{Fe}$ status. It can also take the place of traditional Fe-related biochemical indices that are used by laboratories to detect Fe deficiency and to evaluate the efficacy of Fe supplementation because it is convenient, simple and practical.

Currently, there are few studies investigating sTfR characteristics during Fe supplementation and whether sTfR levels can be used as a reliable indicator for assessing the efficacy of Fe supplementation. In the present study, we implemented a 12-week oral Fe supplementation using Fe-deficient women. We compared the values of laboratory parameters reflecting $\mathrm{Fe}$ status before and after supplementation. There were no dietary restrictions during the supplementation and there were no dietary changes in the women during the study. After Fe supplementation, we found the levels of sTfR significantly decreased in women of childbearing age with IDE and IDA, while the levels of SF significantly increased. In the first 3 weeks, sTfR levels changed little in the IDE group but decreased rapidly in the IDA group. This was probably due to the fact that the Fe deficiency of the IDE group was less severe than that of the IDA group. Furthermore, compared with the change of sTfR levels in the IDA group, its response to Fe supplementation in the IDE group was slower. After 3 weeks, the sTfR levels in both groups decreased progressively and tended to remain steady at week 9 , and reach normal levels at week 12 . This indicated that sTfR levels began to return to normal when the body Fe was gradually supplemented. At week 6, the sTfR levels were close to the normal control group (18.57 (SD 4.48) nmol/l. This means that the body Fe-deficiency status was basically recovered after 6 weeks of Fe supplementation in both the IDE and IDA groups. In the following several weeks of Fe supplementation, the sTfR level still decreased gradually. This was probably due to the increase of $\mathrm{Fe}$-storage compartments that occurred after that of the functional Fe compartments. Brutsaert et al. considered whether STfR concentrations were related to erythropoietic activity and intracellular $\mathrm{Fe}$ requirements ${ }^{15}$; they were sensitive to Fe supplementation, during the improvement of Fe status. A study was performed in IDA patients for 16 weeks. It showed that the changes of sTfR occurred later than those of SF during Fe supplementation. When these two indices were combined, and sTfR:SF was used, its decrease accelerated

Table 4. Dynamic changes of serum transferrin receptor (sTfR):serum ferritin (SF) ratio in subjects with irondeficiency erythropoiesis and iron-deficiency anaemia during iron supplementation

(Mean values and standard deviations)

\begin{tabular}{|c|c|c|c|c|c|c|c|c|c|c|}
\hline \multirow[b]{3}{*}{ Week } & \multicolumn{5}{|c|}{ Fe-deficiency erythropoiesis } & \multicolumn{5}{|c|}{ Fe-deficiency anaemia } \\
\hline & \multirow[b]{2}{*}{$n$} & \multicolumn{2}{|c|}{ STfR:SF } & \multirow[b]{2}{*}{ sTfR† } & \multirow[b]{2}{*}{ sTfR $\ddagger$} & \multirow[b]{2}{*}{$n$} & \multicolumn{2}{|c|}{ STfR:SF } & \multirow[b]{2}{*}{ sTfR† } & \multirow[b]{2}{*}{ sTfR $\ddagger$} \\
\hline & & Mean & SD & & & & Mean & SD & & \\
\hline 0 & 31 & $27 \cdot 21$ & 14.90 & 1.00 & 1.00 & 45 & $42 \cdot 46$ & $29 \cdot 31$ & 1.00 & 1.00 \\
\hline 3 & 31 & $17 \cdot 66^{\star \star}$ & $12 \cdot 78$ & 1.04 & $1 \cdot 04$ & 45 & $20 \cdot 33^{\star \star}$ & 13.49 & 0.51 & 0.51 \\
\hline 6 & 31 & $12 \cdot 47^{\star}$ & $4 \cdot 08$ & 0.71 & 0.69 & 45 & $15 \cdot 26^{\star}$ & $7 \cdot 05$ & 0.32 & 0.64 \\
\hline 9 & 31 & $10 \cdot 66$ & $3 \cdot 28$ & 0.65 & 0.91 & 45 & $11 \cdot 36^{\star}$ & 4.52 & 0.27 & 0.83 \\
\hline 12 & 31 & $10 \cdot 30^{\star}$ & 3.75 & 0.59 & 0.91 & 45 & 11.52 & $4 \cdot 31$ & 0.26 & 0.98 \\
\hline
\end{tabular}

Mean value was significantly different from that for the previous week: ${ }^{\star} P<0.05,{ }^{\star \star} P<0.01$.

$\dagger$ Ratio of values of sTfR index in groups to those at week 0 (relative ratio with fixed base).

$\ddagger$ Ratio of values of STfR index in groups to those of the previous week (link-relative ratio). 


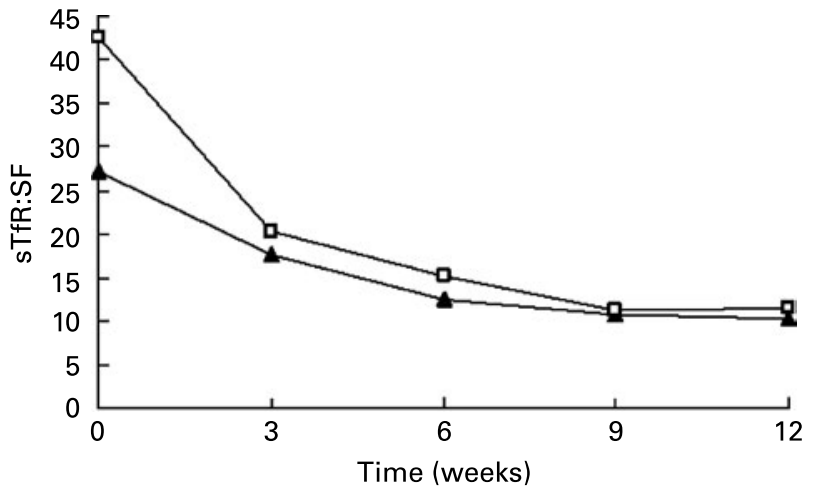

Fig. 2. Values of serum transferrin receptor (sTfR):serum ferritin (SF) ratio in subjects with Fe-deficiency erythropoiesis $(\boldsymbol{\Lambda} ; n 31)$ and Fe-deficiency anaemia $(\square ; n 45)$ during Fe supplementation. Values are means.

along with the administration of Fe. After Fe supplementation, sTfR levels were significantly lower than that before Fe supplementation $^{15}$. Ward et al. considered that SF was the index for evaluating body Fe storage status, STfR was the index for evaluating functional Fe status, and the ratio of sTfR:SF could be used to evaluate body Fe status comprehensively ${ }^{16}$. Skikne et al. found that the value of sTfR:SF was less than 100 when storage $\mathrm{Fe}$ was abundant, while this value was significantly increased with progressive Fe deficiency, reaching as high as 200 when there was severe deficiency of functional $\mathrm{Fe}^{17}$. In the present study, there was no significant difference in SF levels between the IDA stage and the IDE stage. When the sTfR:SF was taken as an observed index, its changing curve was basically parallel with sTfR. This ratio decreased significantly after Fe supplementation in both groups. In the IDE stage, it decreased from 27.21 (SD 14.90) before Fe supplementation to 10.30 (SD 3.75) after Fe supplementation, while it decreased from 42.46 (SD 29.31) to 11.52 (SD 4.31) in the IDA stage. Compared with the change of sTfR in IDA, the decrease of sTfR:SF was more significant during Fe supplementation. It shows that sTfR:SF is also a sensitive index for evaluating the efficacy of Fe supplementation. Because the determination of sTfR levels requires very small $(\mathrm{ml})$ blood samples and requires a very simple analysis, it is more practical in evaluating the efficacy of the intervention.

The present study observed the trend of dynamic changes of sTfR and sTfR:SF during Fe supplementation. It indicates that both can be used as specific indices for determining the efficacy of $\mathrm{Fe}$ supplementation. It provides important evidence for using sTfR as a sensitive index for screening Fe deficiency and assessing the efficacy of $\mathrm{Fe}$ supplementation to alleviate Fe deficiency.

\section{Acknowledgements}

The present study was conducted at the Department of Nutrition and Food Hygiene, School of Public Health, Peking
University, with funding from the Danone Nutrition Institute (38 Xueyuan Road, Beijing, 100083, China).

\section{References}

1. Cook JD (1999) Defining optimal body iron. Proc Nutr Soc 58, 489-495.

2. Breymann C (2002) Iron deficiency and anaemia in pregnancy: modern aspects of diagnosis and therapy. Blood Cells Mol Dis 29, 506-516.

3. Iannotti LL, Tielsch JM, Black MM \& Black RE (2006) Iron supplementation in early childhood: health benefits and risks. Am J Clin Nutr 84, 1261-1276.

4. Piéroni L, Mekhloufi F, Thiolières JM, Hainque B, Herson S \& Jardel C (2005) Soluble transferrin receptor in hemochromatosis patients during phlebotomy therapy. Clin Chim Acta 353, 61-66.

5. Flesland O, Eskelund AK, Flesland AB, Falch D, Solheim BG \& Seghatchian J (2004) Transferrin receptor in serum. A new tool in the diagnosis and prevention of iron deficiency in blood donors. Transfus Apher Sci 31, 11-16.

6. Khalid S, McGrowder D, Kemp M \& Johnson P (2007) The use of soluble transferrin receptor to assess iron deficiency in adults with cystic fibrosis. Clin Chim Acta 378, 194-200.

7. Wang H (1993) Evaluation on validity of iron status parameters identifying iron status. J Clin Intern Med 10, 17-19.

8. Hastka J, Lasserre JJ, Schwarzbeck A \& Hehlmann (1994) Central role of zinc protoporphyrin in staging iron deficiency. Clin Chem 40, 768-773.

9. Ho CH, You JY, Chau WK, Hsu HC, Gau JP, Chen CC \& Yu TJ (2003) Diagnostic value of serum transferrin receptor and glycosylated hemoglobin on hemolytic anemia. Ann Hematol 82, 228-230.

10. Basora M, Deulofeu R, Salazar F, Quinto L \& Gomar C (2006) Improved preoperative iron status assessment by soluble transferrin receptor in elderly patients undergoing knee and hip replacement. Clin Lab Haematol 28, 370-375.

11. Kasvosve I, Gomo ZA, Nathoo KJ, Matibe P, Mudenge B, Loyevsky M, Nekhai S \& Gordeuk VR (2006) Association of serum transferrin receptor concentration with markers of inflammation in Zimbabwean children. Clin Chim Acta 371, 130-136.

12. Kohgo $\mathrm{Y}$, Nishisato $\mathrm{T}$, Kondo $\mathrm{H}$, Tsushima N, Niitsu $\mathrm{Y}$ \& Urushizaki I (1986) Circulating transferrin receptor in human serum. Br J Haematol 64, 277-281.

13. Flowers CH, Skikne BS, Covell AM \& Cook JD (1989) The clinical measurement of serum transferrin receptor. J Lab Clin Med 114, 368-377.

14. Beguin Y, Huebers HA, Josephson B \& Finch CA (1988) Transferrin receptors in rat plasma. Proc Natl Acad Sci U S A 85, 637-640.

15. Brutsaert TD, Hernandez-Cordero S, Rivera J, Viola T, Hughes G \& Haas JD (2003) Iron supplementation improves progressive fatigue resistance during dynamic knee extensor exercise in iron-depleted, nonanemic women. Am J Clin Nutr 77, 441-448.

16. Ward JH, Kushner JP \& Kaplan J (1982) Transferrin receptors of human fibroblasts. Analysis of receptor properties and regulation. Biochem J 208, 19-26.

17. Skikne BS, Flowers CH \& Cook JD (1990) Serum transferring receptor: a quantitative measure of tissue iron deficiency. Blood 75, 1870-1876. 